\title{
Medidas de desempeño en la logística humanitaria: revisión de literatura
}

DOI: $10.46932 / \mathrm{sfjdv} 2 \mathrm{n} 2-105$

Received in: March 1st, 2021

Accepted in: May 30th, 2021

\section{Mg. Juan Camilo López-Vargas}

Estudiante de Doctorado en Ingeniería - Industria y Organizaciones de la Universidad Nacional de Colombia Sede Manizales. Maestría en Ingeniería, Especialización en Dirección de Producción y Operaciones, e Ingeniero Industrial de la Universidad Nacional de Colombia.

Universidad Nacional de Colombia Sede Manizales. Kilómetro 4 Vía al Magdalena, Campus La Nubia, Bloque Q - Piso 2.

Correo: jclopezva@unal.edu.co

\section{Dra. Diana María Cárdenas-Aguirre}

Profesora Asociada del Departamento de Ingeniería Industrial, Facultad de Ingeniería y Arquitectura, Universidad Nacional de Colombia Sede Manizales. Doctora en Ciencias Técnicas del Instituto Superior Politécnico "José Antonio Echeverría”. Especialización en Desarrollo Gerencial de Universidad Autónoma de Manizales, e Ingeniera Industrial de la Universidad Nacional de Colombia. Universidad Nacional de Colombia Sede Manizales. Kilómetro 4 Vía al Magdalena, Campus La Nubia, Bloque Q - Piso 2.

Correo: dmcardenasa@unal.edu.co

\section{RESUMEN}

El propósito de este trabajo es describir y analizar las medidas de desempeño utilizadas en la logística humanitaria. Basados en la importancia de medir el desempeño en los procesos de preparación y atención de desastres, así como en los aportes encontrados en la literatura, se concluye que las medidas de desempeño para estas operaciones pueden enmarcarse en dos objetivos principales: eficiencia y efectividad. No obstante, estos objetivos presentan diferentes prioridades de acuerdo con la fase del ciclo de la gestión de desastres, dado que, en las fases previas al desastre, los propósitos están más enfocados hacia la eficiencia operacional, mientras en las fases posteriores al desastre, los objetivos de efectividad cobran mayor importancia.

Palabras Clave: Medidas de Desempeño, Logística Humanitaria, Preparación y Respuesta a Desastres.

\section{INTRODUCCIÓN}

La gestión logística de las operaciones de atención de desastres, denominada logística humanitaria (Baldini et al., 2012), enmarca todos los procesos relacionados con el aprovisionamiento, almacenamiento, transporte y distribución de bienes, equipos, personas y servicios requeridos para atender a la población afectada por un desastre (Beamon y Balcik, 2008; Day, 2014). La logística humanitaria tiene como objetivo proveer la ayuda humanitaria tan pronto sea posible para reducir el número de muertes y el sufrimiento humano (Balcik y Beamon, 2008; Hasanzadeh y Bashiri, 2016). La gestión de desastres se divide en cuatro etapas sucesivas: mitigación, preparación, respuesta y recuperación (Altay y Green, 2006; Van Wassenhove, 2006). Estas etapas comprenden operaciones de distinta naturaleza, tanto previo 
a la ocurrencia del desastre (mitigación y preparación), como en los momentos posteriores al evento (respuesta y recuperación) (Kovács y Spens, 2007).

La logística humanitaria se enmarca en un contexto bastante complejo: los desastres producen graves afectaciones en la infraestructura y los sistemas de comunicaciones (Baldini et al. 2012; Kumar y Havey, 2013); existe un escenario de alta incertidumbre, dinámico y con poca información confiable (Holguín-Veras et al., 2012); además, se suma la cantidad y diversidad de los actores involucrados, cada uno con distintos objetivos, métodos, capacidades y experiencia logística (Kumar y Havey, 2013; Nikbakhsh y Farahani, 2011; Sebbah et al., 2013).

Teniendo que la logística representa un $80 \%$ de las operaciones de atención de desastres (Van Wassenhove, 2006), medir el desempeño resulta crucial para las organizaciones humanitarias (Beamon y Balcik, 2008). No obstante, la falta de planeación logística puede causar ineficiencias representadas en la deficiente gestión de inventarios, congestión en puertos y la distribución de artículos no requeridos por las comunidades (Charles y Lauras 2011).

Por su parte, el desempeño en las cadenas logísticas humanitarias se ha convertido en un factor clave que permite asegurar la eficiencia y efectividad de las operaciones; sin embargo, la medición del desempeño en este contexto no ha tenido mucha atención en la literatura y está aún en su infancia (Abidi y Scholten, 2015; Beamon y Balcik, 2008). Al respecto, Das (2016) sostiene que el desempeño de las operaciones humanitarias es difícil de calcular, ya que para las organizaciones sin ánimo de lucro no es fácil evaluar la satisfacción de la demanda ni controlar los flujos de dinero y de otros recursos.

Con base en lo anterior, este trabajo se ha diseñado con el propósito de identificar las medidas de desempeño que desde la literatura se definen para la logística humanitaria en función de las etapas previas y posteriores a los desastres.

Este artículo se estructura de la siguiente manera: la sección 2 se encarga de presentar la importancia de medir y evaluar el desempeño en los procesos logísticos en el contexto humanitario, la sección 3 está dedicada a la descripción y análisis de las medidas de desempeño en los procesos de gestión de desastres y, finalmente, se presentan las conclusiones relacionadas con la temática desarrollada.

\section{LA IMPORTANCIA DE MEDIR Y EVALUAR EL DESEMPEÑO EN LOS PROCESOS LOGÍSTICOS}

El ambiente impredecible y de alta incertidumbre generado por un desastre resulta un factor fundamental en el diseño de una cadena logística humanitaria; además, la necesidad de considerar criterios asociados al costo, a los tiempos de respuesta y al nivel de cobertura esperada, hacen del diseño del sistema una labor aún más compleja (Tofighi et al., 2016). Por su parte, Rottkemper et al. (2011) argumentan que 
el proceso de planeación de la acción humanitaria debe considerar dos objetivos en conflicto: la reducción de la demanda insatisfecha a través de la atención efectiva a las comunidades afectadas, y el control de los costos, teniendo que la escasez de los recursos financieros es característica del contexto del desastre. Además, las organizaciones humanitarias presentan serias dificultades para medir el cumplimiento de las necesidades de la población y controlar los flujos de dinero, y en cambio, tienden a medir su desempeño en función de los ingresos o entradas de recursos basados en las donaciones recibidas (Beamon y Balcik, 2008; Das, 2016). Pese a ello, Beamon y Balcik (2008) argumentan que el sector humanitario comienza a demostrar mayor interés en las medidas de desempeño, en virtud de la alta competencia interna del sector por obtener recursos escasos y por mejorar su visibilidad y transparencia.

Desde el enfoque de las cadenas de abastecimiento, un mejor desempeño logístico se obtiene cuando las relaciones entre los actores se gestionan a partir de una perspectiva integral, que permita coordinar las operaciones de manera efectiva y eficiente, eliminando redundancias y aumentando la eficiencia en toda la cadena de abastecimiento humanitaria (Cozzolino, 2012). La importancia de la medición del desempeño en el contexto humanitario se debe a dos factores: primero, por la influencia que tienen la efectividad y la capacidad de respuesta sobre la protección de la vida de las personas; y segundo, por las condiciones de escasez de los recursos para la respuesta a los desastres (Nikbakhsh y Farahani, 2011). En este sentido, el control de las operaciones y el registro de datos e información se ven condicionados por la urgencia y el ritmo de la fase de respuesta, en la que se prioriza la entrega de la ayuda humanitaria tan rápido como sea posible (Das, 2016). Otros aspectos identificados, tienen que ver con la intangibilidad de los servicios ofrecidos y de los resultados obtenidos, así como la complejidad de la misión humanitaria incrementada por el número, variedad e intereses de los actores involucrados (Beamon y Balcik, 2008).

Altay y Green (2006) y Beamon y Balcik (2008) resaltan la necesidad de hacer más énfasis en los modelos de gestión de operaciones de desastres, de implementar nuevas tecnologías y de comprender las características propias de cada evento. Nikbakhsh y Farahani (2011) establecen como línea de investigación el desarrollo de marcos integrales para la medición del desempeño que consideren las características inherentes del contexto del desastre e incorporen los aspectos intangibles de los sistemas logísticos humanitarios. Los aportes que pueden darse desde el sector académico están relacionados con la transferencia del conocimiento e integración con las organizaciones, donde se aproveche la experiencia de los académicos en áreas como la gestión de medidas de desempeño, la gestión de proyectos y el mejoramiento continuo (Thomas y Kopczak, 2005; Van Wassenhove, 2006). 


\section{LA MEDICIÓN DEL DESEMPEÑO EN LOS SISTEMAS LOGÍSTICOS HUMANITARIOS}

Teniendo que el objetivo de la logística humanitaria se centra en la reducción de pérdidas humanas y materiales, el desempeño de la cadena logística humanitaria puede ser medido en función de la capacidad de reacción y la eficiencia y velocidad de la respuesta (Day, 2014). Akhtar et al. (2012) afirman que el desempeño puede determinarse según la cantidad de donaciones entregadas, los tiempos de entrega, los costos de inventario, los costos de transporte, la flexibilidad y la satisfacción de los donantes y beneficiarios.

El factor del costo en el contexto de la logística humanitaria tiene un interés relevante, tal como ocurre en el sector comercial, ya que los recursos económicos son sumamente escasos si se considera que la mayoría de las organizaciones humanitarias se financian principalmente a través de donaciones (Kovács y Spens, 2007). Además, se tiene el costo de la vida humana, el cual está por encima de cualquier otro costo en la respuesta al desastre (Van Wassenhove, 2006), y que puede medirse en función de las pérdidas humanas generadas por la falta de entrega de la ayuda humanitaria a tiempo (Diedrichs et al., 2016).

Pese a sus diferencias, la logística humanitaria considera aspectos del costo que son tradicionales del sector comercial. Diversos modelos encontrados en la literatura incorporan funciones objetivo que persiguen la minimización de los costos de: compras de suministros, localización de instalaciones, preposicionamiento de inventarios, mantenimiento de inventarios, transporte, distribución, y costos totales del sistema (Ver tabla 1). Otras medidas que permiten analizar el desempeño operacional y han sido definidas en la literatura incluyen indicadores como: el tiempo total de transporte y de distribución (Tofighi et al., 2016); el nivel de cobertura y/o demanda insatisfecha (Kunz et al., 2014; Özdamar et al., 2004); la resiliencia, entendida como la capacidad del sistema para retornar a su estado original luego del impacto de un desastre (Hellingrath et al., 2015); y el costo de privación, definido como la valoración económica del sufrimiento de las víctimas causado por la falta de acceso a la ayuda humanitaria (HolguínVeras et al., 2013; Pradhananga et al., 2016).

Las medidas de desempeño descritas pueden clasificarse en dos objetivos fundamentales: la eficiencia y la efectividad. De acuerdo con Beamon y Balcik (2008), mientras la eficiencia mide el uso económico de los recursos, la efectividad define el nivel de cumplimiento de las necesidades de las personas. En la tabla 1, se muestran las medidas de desempeño de eficiencia y efectividad encontradas en la literatura, según las funciones objetivo que los autores consideraron en los modelos propuestos.

Tabla 1. Medidas de desempeño en la logística humanitaria.

\begin{tabular}{|l|l|l|}
\hline Categoría & $\begin{array}{l}\text { Medidas de desempeño } \\
\text { Función objetivo }\end{array}$ & Autores \\
\hline Eficiencia & Costo de transporte / distribución & $\begin{array}{l}\text { Barbarosoğlu y Arda (2004); Davis et al. (2013); Garrido } \\
\text { et al. (2015); Haghani y Oh (1996); Hasanzadeh y Bashiri } \\
\text { (2016); Marcelin et al. (2016); Moreno et al. (2016); }\end{array}$ \\
\hline
\end{tabular}




\begin{tabular}{|c|c|c|}
\hline & & $\begin{array}{l}\text { Rekik et al. (2013); Rezaei-Malek et al. (2016); Sheu } \\
\text { (2007); Wei et al. (2015). }\end{array}$ \\
\hline & $\begin{array}{l}\text { Costo de pre-posicionamiento / } \\
\text { compras / inventario / alistamiento } \\
\text { de almacenes }\end{array}$ & $\begin{array}{l}\text { Caunhye et al. (2016); Davis et al. (2013); Hasanzadeh y } \\
\text { Bashiri (2016); Moreno et al. (2016); Pradhananga et al. } \\
\text { (2016); Rezaei-Malek et al. (2016); Wei et al. (2015). }\end{array}$ \\
\hline & $\begin{array}{l}\text { Costo total de la operación / } \\
\text { respuesta al desastre }\end{array}$ & $\begin{array}{l}\text { Charles y Lauras (2011); Diedrichs et al. (2016); Krejci } \\
\text { (2015); Lorca et al. (2015); Rottkemper et al. (2011); } \\
\text { Tzeng et al. (2007). }\end{array}$ \\
\hline & $\begin{array}{l}\text { Costo de la vida humana / } \\
\text { demanda insatisfecha }\end{array}$ & $\begin{array}{l}\text { Barbarosoğlu y Arda (2004); Diedrichs et al. (2016); } \\
\text { Moreno et al. (2016); Tofighi et al. (2016). }\end{array}$ \\
\hline & $\begin{array}{l}\text { Costo de ruptura de inventarios / } \\
\text { inventarios no utilizados }\end{array}$ & $\begin{array}{l}\text { Davis et al. (2013); Rezaei-Malek et al. (2016); Tofighi } \\
\text { et al. (2016). }\end{array}$ \\
\hline & Costo de localización & Moreno et al. (2016); Sebbah et al. (2013). \\
\hline & Costo de privación & Holguín-Veras et al. (2013); Pradhananga et al. (2016). \\
\hline \multirow{8}{*}{ Efectividad } & $\begin{array}{l}\text { Cobertura / demanda satisfecha / } \\
\text { insatisfecha }\end{array}$ & $\begin{array}{l}\text { Afshar y Haghani (2012); Balcik y Beamon (2008); Davis } \\
\text { et al. (2013); Kunz et al. (2014); Kusumastuti et al. } \\
\text { (2013); Özdamar et al. (2004); Rekik et al. (2013); } \\
\text { Shafiee y Berglund (2016); Sheu (2007); Tzeng et al. } \\
\text { (2007); Yi y Özdamar (2007). }\end{array}$ \\
\hline & $\begin{array}{l}\text { Tiempo de transporte / distribución } \\
\text { / tiempo de llegada de la ayuda / } \\
\text { Lead time }\end{array}$ & $\begin{array}{l}\text { Altay y Pal (2014); Caunhye et al. (2016); Fikar et al. } \\
\text { (2016); Garrido et al. (2015); Kusumastuti et al. (2013); } \\
\text { Özdamar et al. (2004); Rekik et al. (2013); Sahin et al. } \\
\text { (2016); Tofighi et al. (2016); Tzeng et al. (2007); } \\
\text { Xanthopoulos y Koulouriotis (2013). }\end{array}$ \\
\hline & $\begin{array}{l}\text { Número de instalaciones / } \\
\text { almacenes }\end{array}$ & Balcik y Beamon (2008); Rekik et al. (2013). \\
\hline & $\begin{array}{l}\text { Cantidad de ayuda y servicios } \\
\text { entregados }\end{array}$ & Rachaniotis et al. (2013). \\
\hline & $\begin{array}{l}\text { Accesibilidad de la red durante la } \\
\text { respuesta y recuperación }\end{array}$ & Aksu y Ozdamar (2014). \\
\hline & $\begin{array}{l}\text { Cantidad de demanda satisfecha } \\
\text { por unidad de costo }\end{array}$ & Krejci (2015). \\
\hline & $\begin{array}{l}\text { Tiempo esperado para la } \\
\text { recuperación del sistema }\end{array}$ & Krejci (2015); Lorca et al. (2015). \\
\hline & $\begin{array}{l}\text { Tiempo promedio con órdenes } \\
\text { atrasadas }\end{array}$ & rejci (2015). \\
\hline
\end{tabular}

El uso de estos indicadores demuestra que en los últimos tiempos se han adelantado importantes esfuerzos en la incorporación de los conceptos de la logística comercial en la medición del desempeño de las operaciones llevadas a cabo en la logística humanitaria. Las medidas de desempeño resultan diversas, tanto para los indicadores de eficiencia como los de efectividad. A pesar de las diferencias ya explicadas, casi todas las medidas de desempeño provienen del sector comercial. Esto refleja el esfuerzo de los académicos en su afán por aportar instrumentos y mecanismos para determinar el desempeño de la acción humanitaria, a conciencia de las complejidades en las que deben desenvolverse estas organizaciones.

De la tabla 1, se observa que los indicadores de eficiencia de mayor aplicación son el costo de transporte y de distribución, y los costos de inventarios (compras, pre-posicionamiento y mantenimiento). Es importante resaltar el interés por cuantificar las pérdidas y el sufrimiento humano, imponiendo en sus 
modelos variables que castigan su función objetivo cuando las víctimas no son atendidas a tiempo. El desempeño en términos de efectividad puede evaluarse de diversas formas, entre las que se destacan: la cobertura (maximización de la demanda satisfecha o minimización de la demanda insatisfecha) y los tiempos de distribución a las zonas afectadas. Asimismo, se observa una tendencia hacia la formulación de problemas multi-objetivo, ya que los autores evalúan sus modelos a partir de dos o más medidas de desempeño, sea entre indicadores de eficiencia, entre indicadores de efectividad, o una combinación de ambos. Esto concuerda con Garrido et al. (2015) y Shafiee y Berglund (2016), quienes sostienen que las organizaciones deben medir el desempeño no solo desde la función del costo, sino también deben definir sus prioridades hacia una respuesta efectiva de los desastres, es decir, la respuesta no debe realizarse con una perspectiva económica, sino que también deberá ser ágil, equitativa y justa con toda la población afectada.

Por otra parte, resulta conveniente clasificar las medidas de desempeño utilizadas en la literatura de acuerdo con los momentos previos o posteriores al desastre. Con este fin se ha diseñado la tabla 2, que presenta las funciones objetivo tratadas en la tabla 1 según las etapas de la gestión de desastres.

Tabla 2. Medidas de desempeño según las etapas de la logística humanitaria.

\begin{tabular}{|l|l|}
\hline $\begin{array}{l}\text { Etapa del ciclo } \\
\text { del desastre }\end{array}$ & Medidas de desempeño / Función objetivo \\
\hline Preparación & $\begin{array}{l}\text { Costo de transporte } \\
\text { Costo de pre-posicionamiento / inventario / alistamiento de almacenes } \\
\text { Costo de localización } \\
\text { Número de instalaciones / almacenes }\end{array}$ \\
\hline & $\begin{array}{l}\text { Costo de distribución / transporte } \\
\text { Costo de compras } \\
\text { Costo Total del sistema / respuesta al desastre } \\
\text { Costo de la vida humana / demanda insatisfecha } \\
\text { Costo de ruptura de inventarios / inventarios no usados } \\
\text { Respuesta } \\
\text { Costo de localización } \\
\text { Costo de privación } \\
\text { Cobertura / demanda satisfecha / insatisfecha } \\
\text { Tiempo de transporte / distribución / tiempo de llegada de la ayuda / Lead time } \\
\text { Número de instalaciones / almacenes } \\
\text { Cantidad de ayuda y servicios entregados } \\
\text { Accesibilidad de la red durante la respuesta } \\
\text { Cantidad de demanda satisfecha por costos invertidos } \\
\text { Tiempo promedio con órdenes atrasadas }\end{array}$ \\
\hline $\begin{array}{l}\text { Costos de operación } \\
\text { Tiempo de operación } \\
\text { Accesibilidad de la red durante la recuperación } \\
\text { Tiempo esperado para la recuperación del sistema }\end{array}$ \\
\hline
\end{tabular}

En la tabla 2 se observa que la mayor parte de los objetivos se concentra en la etapa de respuesta. Esto redunda en el valor y el interés por evaluar el desempeño logístico en las operaciones durante la atención y respuesta inmediata a los desastres, desde los costos, el tiempo y la cobertura. Por su parte, la 
fase de preparación se caracteriza por contar con indicadores centrados en el costo, mientras que la fase de recuperación ha sido analizada a partir de medidas de desempeño tanto del costo como de la efectividad. Estas observaciones, concuerdan con lo planteado por autores como Caunhye et al. (2016), Hasanzadeh y Bashiri (2016), Rezaei-Malek et al. (2016) y Van Wassenhove (2006), para quienes la prioridad de los objetivos varía de acuerdo con la fase del desastre. Así, durante la preparación, los objetivos están más dirigidos hacia la reducción de costos través del diseño de una cadena eficiente, mientras que, durante la etapa de respuesta, el factor tiempo es fundamental para reducir el sufrimiento de las víctimas, a través de una efectividad basada en la velocidad y la agilidad de la cadena logística. Posteriormente, cuando se inicia la fase de recuperación, se tienen propósitos mixtos, donde persiste la necesidad de una atención efectiva a las comunidades, pero a unos costos razonables.

Cabe mencionar que los indicadores utilizados en la etapa de respuesta no sólo se enfocan a la efectividad, como muestra la tabla 2, sino que otros son propios de la eficiencia, lo cual ofrece a las organizaciones humanitarias una serie de indicadores que les permiten analizar su desempeño en términos tanto de eficiencia como de efectividad, en otras palabras, aunque la importancia de la agilidad en la cadena logística es indiscutible, los costos operacionales no deben descuidarse, máxime el difícil acceso a recursos físicos y monetarios, que el contexto de los desastres implica en la gestión logística de las operaciones.

\section{CONCLUSIONES}

La logística humanitaria, enmarcada en el campo de la gestión de cadenas de abastecimiento, es un área en pleno desarrollo que ha tenido un crecimiento importante durante los últimos años. Esto obedece a que los desastres siguen provocando pérdidas humanas, económicas y ambientales que, con una tendencia incremental de su intensidad y frecuencia, requieren de mayores esfuerzos económicos e investigativos desde los sectores públicos, privados y académicos con el fin de fortalecer la sostenibilidad de los sistemas sociales, económicos, tecnológicos y naturales.

Teniendo en cuenta que existen diferencias sustanciales entre las cadenas logísticas comerciales y las cadenas humanitarias, la literatura ha demostrado que es posible, a pesar de la complejidad del contexto de los desastres, aplicar medidas de desempeño para determinar la eficiencia y la efectividad de las operaciones desarrolladas por los actores involucrados en la preparación y respuesta a los desastres. En este sentido, los indicadores de eficiencia enfocados en la reducción de costos tienen mayor prioridad en la etapa de preparación. Por su parte, durante la respuesta, cobran valor los propósitos de efectividad, concentrando los esfuerzos en mejorar indicadores de agilidad, tiempos de respuesta, calidad de los servicios prestados, y equidad en la cobertura, pero sin descuidar el factor costo, es decir, la respuesta 
debe gestionarse con un abanico de indicadores que cubran tanto la eficiencia como la efectividad operacional. Asimismo, en la fase de recuperación se buscan objetivos de eficiencia y efectividad, procurando que los proyectos de estabilización y reconstrucción de las zonas afectadas se ejecuten con un uso eficiente de los recursos, pero sin descuidar la atención y el seguimiento a las víctimas del desastre.

Por último, la apropiación de las medidas de desempeño por parte de los actores involucrados en las cadenas logísticas humanitarias tiene una importancia fundamental, en la medida que las organizaciones logren asimilar y gestionar estos indicadores entre los demás miembros de la cadena. De esta forma, las organizaciones humanitarias tendrán mayores posibilidades de mejorar el desempeño en los procesos tanto de preparación como de respuesta a los desastres, con el fin de garantizar la sostenibilidad y preservación de la vida de las comunidades más vulnerables. 


\section{REFERENCIAS}

Abidi, H., \& Scholten, K. (2015). Applicability of Performance Measurement Systems to Humanitarian Supply Chains. In Klumpp, M. et al. (eds.). Humanitarian Logistics and Sustainability. New York: Springer, pp. 235-260.

Afshar, A., \& Haghani, A. (2012). Modeling integrated supply chain logistics in real-time large-scale disaster relief operations. Socio-Economic Planning Sciences, Vol. 46, No. 4, pp. 327-338. doi: 10.1016/j.seps.2011.12.003

Akhtar, P., Marr, N. E., \& Garnevska, E. V. (2012). Coordination in humanitarian relief chains: chain coordinators. Journal of Humanitarian Logistics and Supply Chain Management, Vol. 2, No. 1, pp. 85103. doi: $10.1108 / 20426741211226019$

Aksu, D. T., \& Ozdamar, L. (2014). A mathematical model for post-disaster road restoration: Enabling accessibility and evacuation. Transportation Research Part E, Vol. 61, pp. 56-67. doi: 10.1016/j.tre.2013.10.009

Altay, N., \& Green, W. G. (2006). OR/MS research in disaster operations management. European Journal of Operational Research, Vol. 175, No. 1, pp. 475-493. doi: 10.1016/j.ejor.2005.05.016

Altay, N., \& Pal, R. (2014). Information Diffusion among Agents: Implications for Humanitarian Operations. Production and Operations Management, Vol. 23, No. 6, pp. 1015-1027. doi: 10.1111/poms.12102

Balcik, B., \& Beamon, B. M. (2008). Facility location in humanitarian relief. International Journal of Logistics: Research and Applications, Vol. 11, No. 2, pp. 101-121. doi: 10.1080/13675560701561789

Baldini, G., Oliveri, F., Braun, M., Seuschek, H., \& Hess, E. (2012). Securing disaster supply chains with cryptography enhanced RFID. Disaster Prevention and Management: An International Journal, Vol. 21, No. 1, pp. 51-70. doi: 10.1108/09653561211202700

Barbarosoğlu, G., \& Arda, Y. (2004). A two-stage stochastic programming framework for transportation planning in disaster response. Journal of the Operational Research Society, Vol. 55, No. 1, pp. 43-53. doi: 10.1057/palgrave.jors.2601652

Beamon, B. M., \& Balcik, B. (2008). Performance measurement in humanitarian relief chains. International Journal of Public Sector Management, Vol. 21, No. 1, pp. 4-25. doi: $10.1108 / 09513550810846087$

Caunhye, A. M., Zhang, Y., Li, M., \& Nie, X. (2016). A location-routing model for prepositioning and distributing emergency supplies. Transportation Research Part E, Vol. 90, pp. 161-176. doi: 10.1016/j.tre.2015.10.011

Charles, A., \& Lauras, M. (2011). An enterprise modelling approach for better optimisation modelling: application to the humanitarian relief chain coordination problem. OR Spectrum, Vol. 33, No. 3, pp. 815841. doi: 10.1007/s00291-011-0255-2

Cozzolino, A. (2012). Humanitarian Logistics: Cross-Sector Cooperation in Disaster Relief Management. New York: Springer. 
Das, L. (2016). Role of Humanitarian Supply Chain Management in Various Disaster Situations Across the Globe. In Sahay, B. S. et al. (eds.). Managing Humanitarian Logistics. New York: Springer, pp. 253271.

Davis, L. B., Samanlioglu, F., Qu, X., \& Root, S. (2013). Inventory planning and coordination in disaster relief efforts. International Journal of Production Economics, Vol. 141, No. 2, pp. 561-573. doi: 10.1016/j.ijpe.2012.09.012

Day, J. M. (2014). Fostering emergent resilience: the complex adaptive supply network of disaster relief. International Journal of Production Research, Vol. 52, No. 7, pp. 1970-1988. doi: 10.1080/00207543.2013.787496

Diedrichs, D. R., Phelps, K., \& Isihara, P. A. (2016). Quantifying communication effects in disaster response logistics: A multiple network system dynamics model. Journal of Humanitarian Logistics and Supply Chain Management, Vol. 6, No. 1, pp. 24-45. doi: 10.1108/JHLSCM-09-2014-0031

Fikar, C., Gronalt, M., \& Hirsch, P. (2016). A decision support system for coordinated disaster relief distribution. Expert Systems With Applications, Vol. 57, pp. 104-116. doi: 10.1016/j.eswa.2016.03.039

Garrido, R. A., Lamas, P., \& Pino, F. J. (2015). A stochastic programming approach for floods emergency logistics. Transportation Research Part E-Logistics and Transportation Review, Vol. 75, pp. 18-31. doi: 10.1016/j.tre.2014.12.002

Haghani, A., \& Oh, S. C. (1996). Formulation and Solution of a Multi-Commodity, Multi-Modal Network Flow Model for Disaster Relief Operations. Transportation Research Part A, Vol. 30, No. 3, pp. 231-250. doi: 10.1016/0965-8564(95)00020-8

Hasanzadeh, H., \& Bashiri, M. (2016). An efficient network for disaster management: Model and solution. Applied Mathematical Modelling, Vol. 40, No. 5-6, pp. 3688-3702. doi: 10.1016/j.apm.2015.09.113

Hellingrath, B., Babun, T. A., Smith, J. F., \& Link, D. (2015). Disaster Management Capacity Building at Airports and Seaports. In Klumpp, M. et al. (eds.). Humanitarian Logistics and Sustainability. New York: Springer, pp. 87-112.

Holguín-Veras, J., Jaller, M., Van Wassenhove, L. N., Pérez, N., \& Wachtendorf, T. (2012). On the unique features of post-disaster humanitarian logistics. Journal of Operations Management, Vol. 30, No. 7-8, pp. 494-506. doi: 10.1016/j.jom.2012.08.003

Holguín-Veras, J., Pérez, N., Jaller, M., Van Wassenhove, L. N., \& Aros-Vera, F. (2013). On the appropriate objective function for post-disaster humanitarian logistics models. Journal of Operations Management, Vol. 31, No. 5, pp. 262-280. doi: 10.1016/j.jom.2013.06.002

Kovács, G., \& Spens, K. M. (2007). Humanitarian logistics in disaster relief operations. International Journal of Physical Distribution \& Logistics Management, Vol. 37, No. 2, pp. 99-114. doi: 10.1108/09600030710734820

Krejci, C. C. (2015). Hybrid simulation modeling for humanitarian relief chain coordination. Journal of Humanitarian Logistics and Supply Chain Management, Vol. 5, No. 3, pp. 325-347. doi: 10.1108/JHLSCM-07-2015-0033 
Kumar, S., \& Havey, T. (2013). Before and after disaster strikes: A relief supply chain decision support framework. International Journal of Production Economics, Vol. 145, No. 2, pp. 613-629. doi: 10.1016/j.ijpe.2013.05.016

Kunz, N., Reiner, G., \& Gold, S. (2014). Investing in disaster management capabilities versus prepositioning inventory: A new approach to disaster preparedness, International Journal of Production Economics, Vol. 157, pp. 261-272. doi: 10.1016/j.ijpe.2013.11.002

Kusumastuti, R. D., Wibowo, S. S., \& Insanita, R. (2013). Modeling Facility Locations for Relief Logistics in Indonesia. In Zeimpekis, V. et al. (eds.). Humanitarian and Relief Logistics: Research Issues, Case Studies and Future Trends. New York: Springer, pp. 183-205.

Lorca, Á., Çelik, M., Ergun, Ö., \& Keskinocak, P. (2015). A decision-support tool for post-disaster debris operations. Procedia Engineering, Vol. 107, pp. 154-167. doi: 10.1016/j.proeng.2015.06.069

Marcelin, J. M., Horner, M. W., Ozguven, E. E., \& Kocatepe, A. (2016). How does accessibility to postdisaster relief compare between the aging and the general population? A spatial network optimization analysis of hurricane relief facility locations. International Journal of Disaster Risk Reduction, Vol. 15, pp. 61-72. doi: 10.1016/j.ijdrr.2015.12.006

Moreno, A., Alem, D., \& Ferreira, D. (2016). Heuristic approaches for the multiperiod locationtransportation problem with reuse of vehicles in emergency logistics. Computers \& Operations Research, Vol. 69, pp. 79-96. doi: 10.1016/j.cor.2015.12.002

Nikbakhsh, E., \& Farahani, R. Z. (2011). Humanitarian Logistics Planning in Disaster Relief Operations. In Farahani, R. Z. et al. (eds.). Logistics Operations and Management: Concepts and Models. Amsterdam: Elsevier, pp. 291-332.

Özdamar, L., Ekinci, E., \& Küçükyazici, B. (2004). Emergency Logistics Planning in Natural Disasters. Annals of Operations Research, Vol. 129, No. 1-4, pp. 217-245. doi: 10.1023/B:ANOR.0000030690.27939.39

Pradhananga, R., Mutlu, F., Pokharel, S., Holguín-Veras, J., \& Seth, D. (2016). An integrated resource allocation and distribution model for pre-disaster planning. Computers \& Industrial Engineering, Vol. 91, pp. 229-238. doi: 10.1016/j.cie.2015.11.010

Rachaniotis, N. P., Dasaklis, T., Pappis, C. P., \& Van Wassenhove, L. N. (2013). Multiple Location and Routing Models in Humanitarian Logistics. In Zeimpekis, V. et al. (eds.). Humanitarian and Relief Logistics: Research Issues, Case Studies and Future Trends. New York: Springer, pp. 43-57.

Rekik, M., Ruiz, A., Renaud, J., Berkoune, D., \& Paquet, S. (2013). A Decision Support System for Humanitarian Network Design and Distribution Operations. In Zeimpekis, V. et al. (eds.). Humanitarian and Relief Logistics: Research Issues, Case Studies and Future Trends. New York: Springer, pp. 1-20.

Rezaei-Malek, M., Tavakkoli-Moghaddam, R., Zahiri, B., \& Bozorgi-Amiri, A. (2016). An interactive approach for designing a robust disaster relief logistics network with perishable commodities. Computers \& Industrial Engineering, Vol. 94, pp. 201-215. doi: 10.1016/j.cie.2016.01.014 
Rottkemper, B., Fischer, K., Blecken, A., \& Danne, C. (2011). Inventory relocation for overlapping disaster settings in humanitarian operations. OR Spectrum, Vol. 33, No. 3, pp. 721-749. doi: 10.1007/s00291-011-0260-5

Sahin, H., Kara, B. Y., \& Karasan, O. E. (2016). Debris removal during disaster response: A case for Turkey. Socio-Economic Planning Sciences, Vol. 53, pp. 49-59. doi: 10.1016/j.seps.2015.10.003

Sebbah, S., Boukhtouta, A., Berger, J., \& Ghanmi, A. (2013). Military Logistics Planning in Humanitarian Relief Operations. In Zeimpekis, V. et al. (eds.). Humanitarian and Relief Logistics: Research Issues, Case Studies and Future Trends. New York: Springer, pp. 77-110.

Shafiee, M. E., \& Berglund, E. Z. (2016). Agent-based modeling and evolutionary computation for disseminating public advisories about hazardous material emergencies. Computers, Environment and Urban Systems, Vol. 57, pp. 12-25. doi: 10.1016/j.compenvurbsys.2016.01.001

Sheu, J. B. (2007). An emergency logistics distribution approach for quick response to urgent relief demand in disasters. Transportation Research Part E, Vol. 43, No. 6, pp. 687-709. doi: 10.1016/j.tre.2006.04.004

Thomas, A., \& Kopczak, L. (2005). From logistics to supply chain management: The path forward in the humanitarian sector, white paper, Fritz Institute, San Francisco, CA.

Tofighi, S., Torabi, S. A., \& Mansouri, S. A. (2016). Humanitarian logistics network design under mixed uncertainty. European Journal of Operational Research, Vol. 250, No. 1, pp. 239-250. doi: 10.1016/j.ejor.2015.08.059

Tzeng, G. H., Cheng, H. J., \& Huang, T. D. (2007). Multi-objective optimal planning for designing relief delivery systems. Transportation Research Part E-Logistics and Transportation Review, Vol. 43, No. 6, pp. 673-686. doi: 10.1016/j.tre.2006.10.012

Van Wassenhove, L. N. (2006). Humanitarian aid logistics: supply chain management in high gear. Journal of the Operational Research Society, Vol. 57, No. 5, pp. 475-489. doi: 10.1057/palgrave.jors.2602125

Wei, X., Al-Refaie, A., Robles, M., \& Noche, B. (2015). A Sustainable Humanitarian Relief Network Study for the Wenchuan Earthquake. In Klumpp, M. et al. (eds.). Humanitarian Logistics and Sustainability. New York: Springer, pp. 193-213.

Xanthopoulos, A. S., \& Koulouriotis, D. E. (2013). A Multi-agent Based Framework for Vehicle Routing in Relief Delivery Systems. In Zeimpekis, V. et al. (eds.). Humanitarian and Relief Logistics: Research Issues, Case Studies and Future Trends. New York: Springer, pp. 167-182.

Yi, W., \& Özdamar, L. (2007). A dynamic logistics coordination model for evacuation and support in disaster response activities. European Journal of Operational Research, Vol. 179, No. 3, pp. 1177-1193. doi: 10.1016/j.ejor.2005.03.077 